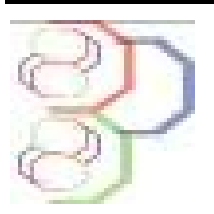

\title{
Diversité floristique et déterminants de l'enherbement des bananeraies industrielles de Dabou au sud de la Côte d'ivoire
}

\author{
Prosper Yao Kouadio*, Marie-Solange Tiébré, Justin N'Dja Kassi, Edouard Kouakou N'Guessan \\ Laboratoire de Botanique, U.F.R. Biosciences, Université Félix Houphouët-Boigny, 22 BP 582 Abidjan 22 Côte d'Ivoire \\ *Author for corresponding: k_yao_pros@yahoo.fr \\ Original submitted in on 17 $7^{\text {th }}$ May 2013 Published online at www.m.elewa.org on $2^{\text {nd }}$ September 2013. \\ https://dx.doi.org/10.4314/jab.v68i0.95066
}

\section{RESUME}

Objectifs : L'étude de la flore adventice des bananeraies de Dabou répond à la recherche de la compétitivité de la banane de Côte d'Ivoire. L'objectif est de connaître les déterminants de l'enherbement pour une production durable de la banane sans herbicides.

Méthodes et résultats : Des paramètres phytosociologiques et agronomiques ont été conjointement suivis dans 4 bananeraies industrielles. Les données obtenues ont été analysées. La flore adventice est très diversifiée et dominée par huit espèces héliophiles majeures. Le non respect du calendrier de désherbage chimique, la nature des sols, la position topographique des parcelles, la faible densité des bananiers et leur faible recouvrement sont les causes de l'enherbement.

Conclusions: Les facteurs phytoécologiques sont les principaux responsables de l'enherbement. La lutte chimique est limitée. La lutte biologique par les Légumineuses de couverture peut être envisagée.

Mots clés : Adventice, Bananier Grande Naine, Côte d'Ivoire, Phytosociologie.

Floral diversity and ecological factors of weediness in the banana industry in Dabou, south of Côte d'Ivoire

\section{ABSTRACT}

Objectives: The objective is to understand the determinants of weed for sustainable banana production without use of herbicides.

Methodology and results: Phytosociological and agronomic parameters were jointly monitored in four bananas industries. The data obtained were analyzed. The flora is diversified and dominated by eight major heliophilous species. Failure in timing of chemical weed control, soil type, plots topographic position, low density of banana and low recovery causes the weed.

Conclusions: Phytoecological factors are primarily responsible for weed. Chemical control is limited. Biological control by Legumes coverage can be considered.

Keywords : Côte d'Ivoire, Grand Nain Banana, Phytosociology

\section{INTRODUCTION}


La banane est un aliment complet qui bénéficie d'une valeur marchande relativement importante et fait l'objet d'échanges commerciaux intenses à travers le monde (M.A.E. et al., 2006). La banane est le principal fruit à faire l'objet d'échanges internationaux et également le plus populaire à travers le monde. Elle est le premier fruit exporté en termes de volume, bien qu'elles ne se placent qu'à la seconde place derrière les agrumes en termes de valeur. La banane est un produit de base très sensible aussi bien d'un point de vue économique, que social, environnemental et politique. Après le riz, le blé_et le maïs, la banane est l'une des plus importantes cultures vivrières du monde. La production mondiale de la banane est de 105 millions de tonnes dans le monde, soit environ 3330 kilos de bananes par seconde. L'Inde est le principal producteur avec $21 \%$ de la production mondiale qu'elle consomme quasi-intégralement. Viennent ensuite le Brésil ( $9 \%$ de la production mondiale), la Chine (9\%), les Philippines (9\%), l'Equateur (8\%) et I'Indonésie (7 \%).Selon la FAO, le montant annuel total des exportations mondiales de banane s'est élevé à 5,8 milliards de dollars en 2006 et représente, de toute évidence, une source vitale de revenus pour de nombreux pays. Aux niveaux local et régional, il existe une relation étroite entre le revenu dégagé de l'industrie de la banane et la sécurité alimentaire des ménages. L'amplitude des quantités exportées ou celle des prix à l'exportation peut être la cause de fluctuations des revenus des personnes directement employées dans le secteur de la banane, qu'il s'agisse des petits exploitants agricoles ou des salariés des plantations. En outre, les industries secondaires et tertiaires, ainsi que leur personnel ressentent également les effets de ces

\section{MATERIELS ET METHODES}

Zone d'étude: L'étude s'est déroulée dans 4 bananeraies regroupées en trois sites (Site 40 à SongonAgban, Site 66: à Songon- M'brathé et le Site 50 regroupant les plantations: d'Agnéby et de N'gatty) de la Compagnie de Développement des Bananes de Côte $d^{\prime \prime}$ Ivoire (CDBCl). Ces plantations sont situées entre $5^{\circ} 39^{\prime}$ et $42^{\circ} 00^{\prime}$ de latitude Nord et entre $4^{\circ} 09^{\prime}$ et $48^{\circ} 00^{\prime}$ de longitude Ouest (Figure 1). Elles s'étendent sur 350 variations (Planetoscope.com, 2012). En Côte d'Ivoire, cette culture offre 10000 emplois pour une superficie de 6000 ha et une production de 294641 tonnes par an (Adé, 2006 ; M.E.F., 2007abc; BCEAO, 2008). Ce secteur contribue pour $8 \%$ au P.I.B. agricole et pour $2 \%$ au P.I.B. national (Adé, 2006).La culture industrielle du bananier fait usage d'une grande quantité de produits chimiques (insecticides, herbicides et fongicides) efficaces contre les maladies et les ravageurs des bananiers mais qui occasionnent des cas de résistances et de maladies au niveau des travailleurs et des consommateurs de la banane. La tendance actuelle est de produire des fruits respectant les normes de qualité en termes de réduction de Limite Maximale de Résidus (L.M.R.) de pesticides et la préservation de l'environnement vis-à-vis de ces produits chimiques. Dans ce cadre, le référentiel de production et de certification (BAGAP) en cours aura pour objectif de réduire de moitié la quantité de pesticides utilisée d'ici 2013 ; l'objectif à terme étant de produire de la banane biologique par l'amélioration des pratiques culturales et l'emploi de variétés alternatives non O.G.M. (Farcey, 2008). La présente étude se propose d'identifier la flore adventice de la culture de bananiers et de déterminer les principaux facteurs responsables de l'enherbement en vue de trouver d'autres alternatives aux désherbages chimiques et mécaniques, coûteux, fastidieux et sources de maladies telles que le cancer, les allergies, les troubles neurologiques et respiratoires, la stérilité, la diminution des défenses immunitaires, les altérations génétiques, etc. (Goldsmith et al., 1990). Les travaux ont été conduits en milieu industriel dans les bananeraies de la zone de Dabou, en basse Côte d'Ivoire.

hectares entre la Commune de Songon et le Département de Dabou au Sud de la Côte d'Ivoire, en bordure de la lagune Ebrié. La région appartient au secteur ombrophile du domaine Guinéen caractérisée par un climat de type sub-équatorial chaud (Température moyenne de $26,3^{\circ} \mathrm{C}$ ) et humide avec une pluviométrie moyenne annuelle $1557 \mathrm{~mm}$ (Avenard, et al.1971). 


\section{Kouadio et al. J. Appl. Biosci. 2013. Diversité floristique et déterminants de l'enherbement des bananeraies industrielles de Dabou, Côte d'Ivoire}

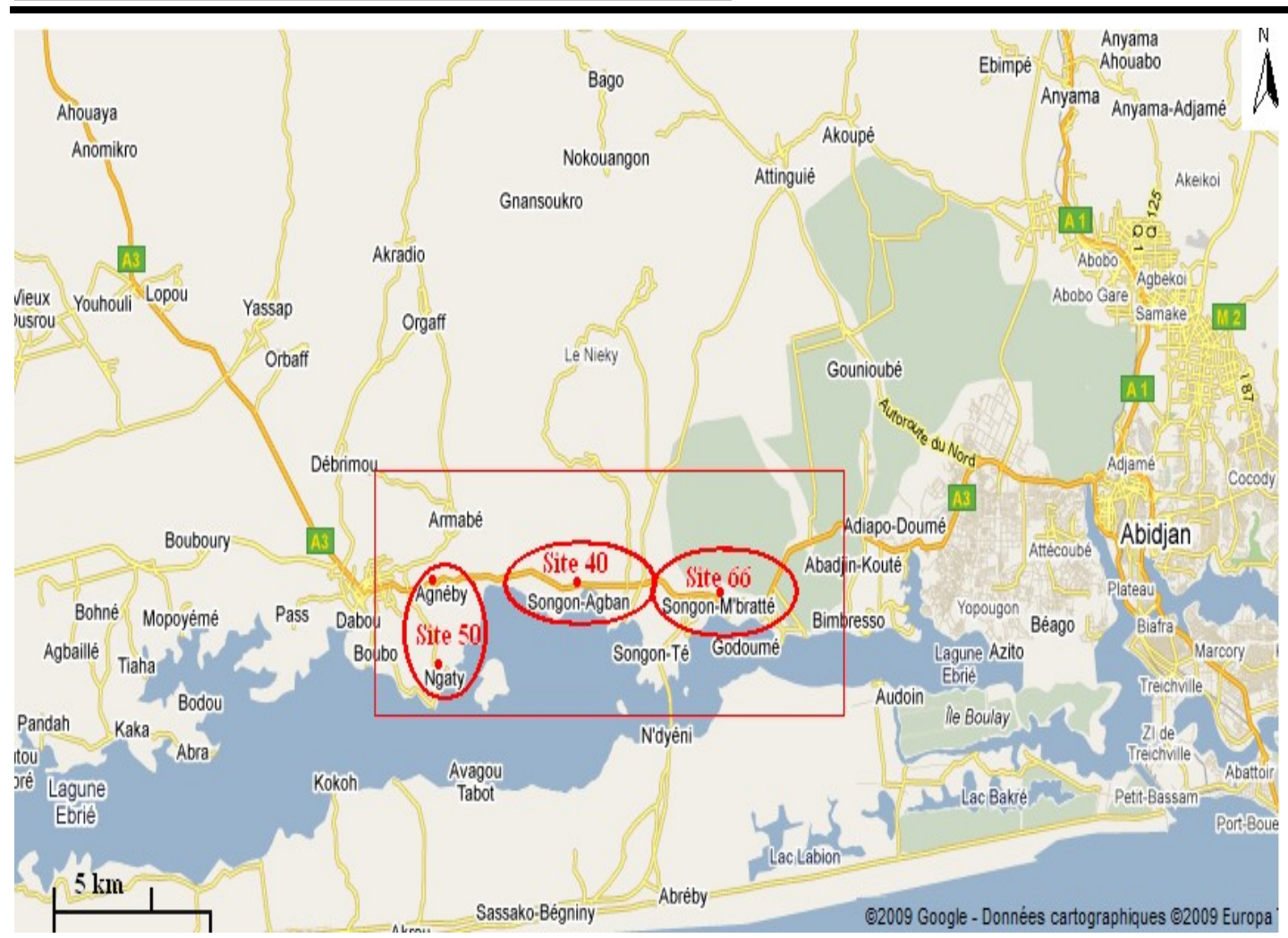

Figure 1-- Localisation des sites de la Compagnie des Bananes de Côte d'Ivoire (CDBCl)

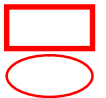
Zone d'étude
Codes des sites (3) selon l'Organisation Centrale de la Commercialisation de l'ananas et de la Banane

Plantations de bananiers (4)

Matériel: Le matériel végétal est constitué des différentes espèces de mauvaises herbes et des bananiers de la variété Grande Naine. Quatre piquets de bambou (Bambusa vulgaris Shrad. Ex J.C. Wendl, Poaceae), des ficelles en plastique, un décamètre, une fiche de relevés phytosociologiques, des fardes de papier journal, un four et un herbarium ont été utilisés lors de cette étude. Les spécimens ont été identifiés au Centre National de Floristique (CNF). Les données floristiques ont été saisies sur Word 2003 et Excel 2003. Les analyses en composantes principales (ACP) ont été faites avec Xlstat 2008.

Méthodes: Des études phytosociologiques et phytoécologiques ont été entreprises dans le cadre de cette étude. La Phytosociologie est la science qui étudie les associations végétales. Elle procède par échantillonnage. Cette méthode a permis d'étudier de manière simultanée, la flore adventice des quatre plantations de bananiers (Songon-Agban, SongonM'brathé, Agnéby et N'gatty) entre le 5 mars 2006 et le 21 novembre 2006. L'étude a débuté par une enquête préliminaire auprès des travailleurs et par des visites sur le terrain qui ont permis d'identifier les paramètres, de définir la taille de l'échantillon et l'aire minimale de $84 \mathrm{~m}^{2}$ (12 $\mathrm{m} \times 7$ ). Cette surface d'échantillonnage élémentaire est obtenue selon la méthode de Boraud (2000) et lpou (2005). Les méthodes de relevé de surface et de relevés itinérants ont été utilisées. Le deuxième type de relevé est utilisé par plusieurs auteurs (Maillet, 1981 ; Loudyi, 1985 ; Chicouène, 2000 ; Le Bourgeois et al., 2004, Le breton et Le Bourgeois, 2005; Touré, 2009). La population étudiée est constituée par l'ensemble des parcelles de bananiers de chaque site. L'échantillonnage est strictement aléatoire. II a consisté à faire des relevés à intervalles réguliers de deux planches de bananiers, à partir de la deuxième planche au niveau de chaque parcelle. Nous avons par exemple échantillonné sur les planches $2 ; 5$ et 8 de dimensions $10 \mathrm{~m} \times 100 \mathrm{~m}$ (1000 $\mathrm{m}^{2}$ ) sur les parcelles (Figure 2). Les relevés sont réalisés sur chacune de ces planches. Les placettes sont espacées de $20 \mathrm{~m}$ et mesurent $12 \mathrm{~m} \times 7 \mathrm{~m}\left(84 \mathrm{~m}^{2}\right)$. Pour obéir au principe de représentativité et de comparabilité que requiert un bon échantillonnage en phytosociologie, le nombre d'observations à réaliser, par parcelle a été fixé d'avance proportionnellement à sa superficie. Ainsi, 6 échantillons sont choisis au niveau des parcelles ayant 
une surface inférieure à 3 hectares et un nombre d'échantillons $=3 \times$ Superficie +1 pour les parcelles dont la superficie est plus grande, Sur cette base, le nombre d'échantillons réalisés est compris entre 10 et 16 par parcelle de bananiers, quel que soit le site. En tout, 592 relevés ont été effectués sur une superficie totale de 259,21 hectares. Les variables floristiques, agrotechniques et naturelles ont été mesurées.

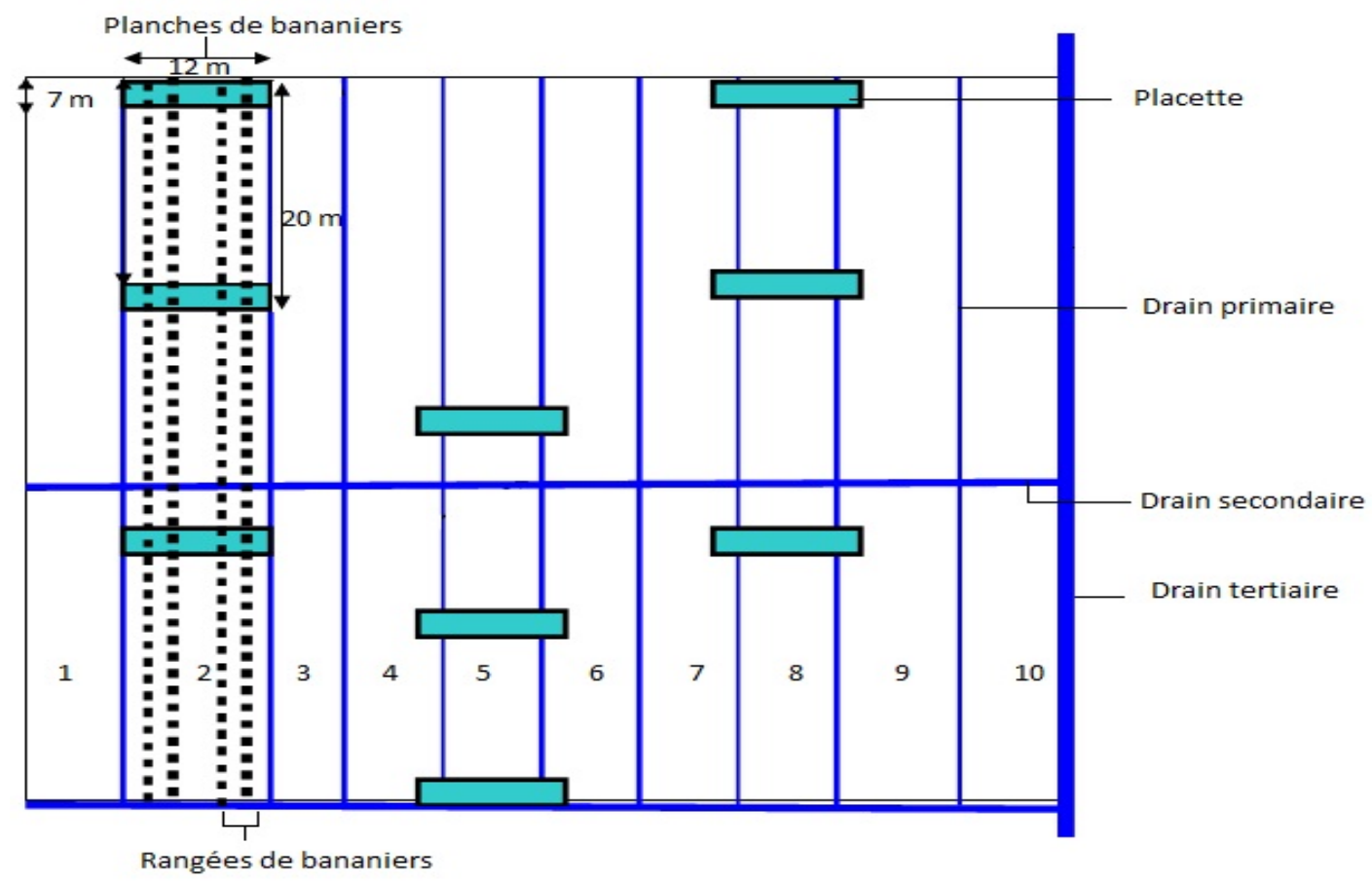

Figure 2-- Dispositif d'échantillonnage au cours des séances de relevés phytosociologiques.
$\square$ Drains (canaux de drainage des eaux)
$1 ; 2 ; 3 ; \ldots 10$ Planches de bananiers
. . . . Rangées de bananiers

Les paramètres floristiques sont : la richesse spécifique et générique telle que définie par Letouzey (1985), le stade phénologique, le type biologique selon Lebrun (1966) et Aké-Assi $(2001$; 2002) tirées de Raunkiaer (1905), la chorologie, la spéciation comme définie par Aké-Assi (2001), le cœfficient de similitude calculé selon la formule de Sørensen (1948) et Gounot (1969). La contribution floristique est évaluée suivant la loi de Daget et Poissonnet (1969), la fréquence relative moyenne est calculée par la formule de Godron (1968) et permet d'établir des classes de fréquences selon Raunkiaer (1934), le coefficient de contribution spécifique a également été calculée par la formule de Daget et Poissonnet (1969) et permet d'établir le niveau d'agressivité d'une espèce par rapport à la culture. Ainsi, Daget et Poissonnet (1969) et Aman (1978) établissent des classes de coefficient de Contribution Spécifique Floristique (CSF (e)) des adventices en fonction de la valeur de leur $\operatorname{CSF}(e)$ :
- $\operatorname{CSF}(e)<1$ : espèces non productrices dont l'effet dépressif sur la culture est plus ou moins négligeable ; $-1<\operatorname{CSF}(e)<4$ : espèces non productrices dont l'effet dépressif sur la culture est relativement élevé, elles sont dites agressives;

- $\operatorname{CSF}(e) \geq 4$ : espèces très productrices dont l'effet dépressif sur la culture est particulièrement élevé ;

L'indice d'abondance-dominance est calculé et permet de savoir si les différents individus d'une espèce sont isolés, regroupés ou dispersés, nombreux, peu ou rares (Guinochet, 1973). L'échelle choisie est l'échelle des indices d'abondance-dominance selon Le Bourgeois (1993) qui est l'échelle modifiée de Braun-Blanquet (1932) :

+ : Espèce représentée par quelques individus épars et peu recouvrant ;

1 : Espèce à densité faible, recouvrant moins de $5 \%$ de la surface échantillonnée ; 
2 : Espèce assez abondante, recouvrant 5 à $25 \%$ de la surface échantillonnée ;

3 : Espèce assez abondante, recouvrant 25 à $50 \%$ de la surface échantillonnée ;

4 : Espèce abondante, recouvrant plus de $50 \%$ de la surface échantillonnée ;

Les catégories de mauvaises herbes sont définies et traduisent le degré d'infestation des espèces c'est-à-dire leurs fréquences et leurs indices d'abondance-dominance moyenne sur l'ensemble des sites étudiés. Elles sont fonction de la fréquence absolue et du recouvrement global (Marnotte, 1989 ; Traoré, 1991) et sont présentées en 7 groupes d'adventices (N.B. : frm fréquence relative moyenne; IADm Indice d'Abondance-Dominance moyenne):

- les adventices majeures de la zone : frm $>50$ $\%,|\mathrm{ADm} 2<| \mathrm{ADm} \leq 4$ sur la zone ;

- les adventices majeures locales : frm $>50 \%, 2$ $<\mathrm{IADm} \leq 4$ sur le site ;

- les adventices majeures potentielles de la zone : $24 \leq$ frm $\leq 50 \%$;

- $\quad$ AADm $1<\mid A D m \leq 2$; sur la zone ;

- les adventices majeurs potentiels locaux : $24 \leq$ Frm $\leq 50 \%$. ; IADm $1<\mid A D m \leq 3$ sur le site ;

- les adventices accompagnatrices de la zone 10 $\leq$ Frm $\leq 24 \%$;; IADm $\leq 1$ sur la zone ;

- les adventices accompagnatrices du site $10 \leq$ Frm $\leq 24 \%$. ; IADm $\leq 2$ sur le site ;

- les adventices mineures ou accidentelles $10<$ Frm, $+\leq \mathrm{IADm} \leq 4$ sur le site ;

Enfin le recouvrement global des adventices et des bananiers au sens de Gounot (1969) est estimé. Les variables agrotechniques sont la superficie (Sup), le nombre d'échantillons (NE); la position dans la toposéquence (Pt), le type de sol (Sol), le nombre de bananiers (Nbp), le cycle (Cyc), l' âge de la parcelle (Age), le temps entre deux traitements herbicides successifs $(\mathrm{TaH})$, le temps entre la date d'observation et

\section{RESULTATS}

\section{Études phytosociologiques}

Diversité spécifique : La flore des bananeraies étudiées comprend 281 espèces d'adventices reparties entre 222 genres et 83 familles. Les Dicotylédones sont les plus nombreuses (68,3\% des espèces recensées). Cette classe renferme 64 familles, 165 genres et 199 espèces adventices. Les Monocotylédones sont représentées par 8 familles, 43 genres et 67 espèces. Huit familles de Bryophytes sont également présentes et se répartissent entre 10 genres et 11 espèces. Cette classe représente la date d'application herbicide (Tao), le recouvrement des bananiers (Recb), le taux de bananiers coupés (Cpe), le nombre d'espèces adventices (NEA), le recouvrement des adventices (Reca), le nombre d'espèces adventices par placette (Nes / p), la contribution floristique (C.F), l'indice d'abondance-dominance global des adventices (I.a.g), la fréquence relative moyenne des adventices (Frm) et la contribution floristique spécifique moyenne des adventices (CFS). Deux variables naturelles se rapportent au type de sol et à la position topographique de la parcelle étudiée. D'autres variables additionnelles comme le type de paillage des planches de bananiers, la chute de bananiers, les récoltes précoces, observables au moment de l'échantillonnage, ont été prises en compte.

Analyses statistiques: Les méthodes d'analyses multivariées utilisées dans les études floristiques sont les Analyses Factorielles de Correspondances (AFC) et les Analyses en Composantes Principales (ACP) (Guinochet, 1973 ; Le Bourgeois, 1993 ; Boraud, 2000; Touré, 2009). Dans le cadre de cette étude, l'ACP a été retenue pour l'analyse des données. Elle a porté sur les 18 variables obtenues à partir des variables naturelles (2), floristiques (5), agrotechniques (8) et phytosociologiques (3). Elle a été réalisée par plantation, par site et sur la zone de Dabou. La majorité des variables étant numériques (16 variables), les paramètres qualitatifs ont été codées en valeurs numériques. Ce qui a permis d'établir le niveau d'explication entre les descripteurs du milieu et les variables des mauvaises herbes sur les parcelles, les sites et sur la zone étudiée. Ainsi, par cette méthode, il a été possible d'identifier les facteurs écologiques les plus déterminants de l'enherbement des parcelles de bananiers à partir de la distribution dans les différents plans factoriels et de l'analyse graphique représentant, les corrélations entre variables du milieu et celles des adventices avec les axes factoriels.

$3,6 \%$ de la flore adventice. Elle devance de peu les Ptéridophytes qui sont représentées par 3 familles botaniques regroupant 4 genres et 4 espèces. Cette dernière classe constitue $1,3 \%$ de la flore des bananeraies étudiées. Les Poaceae sont de loin la famille la plus présente dans cette flore $(12,1 \%)$. Elles sont suivies des Cyperaceae (7\%), des Euphorbiaceae (7\%), des Rubiaceae (5,7\%) et des Asteraceae (4,6\%). Une autre particularité de cette flore est que les familles sont souvent représentées par 2 espèces végétales au plus. 
Par ailleurs, un nombre relativement élevé d'espèces de champignons, de mousses et d'algues, non identifiées et évoluant sous les bananiers, poussent sur divers substrats (organes végétaux en décomposition, fumier, sol). Les espèces végétales les plus fréquentes, les plus abondantes et les plus agressives vis-à-vis des bananiers sont Phyllanthus urinaria L. (Euphorbiaceae), Cleome ciliata Schum.\&Thonn. (Capparidaceae), Spermacoce latifolia Aubl. (Rubiaceae), Ageratum conyzoides L. (Asteraceae), Mariscus cylindristachyus Steudel (Cyperaceae) et Cyperus sphacelathus Rottb. (Cyperaceae). Au niveau phénologique, les adventices présentent différents stades de développement, au moment des traitements herbicides. Elles sont généralement en floraison ou au stade de la maturité des graines ou de déhiscence des fruits. Quelques espèces sont au stade de jeunes plants (Talinum triangulare (Jacq.) Willd. (Portulacaceae), Portulaca oleracea L. (Portulacaceae), Calopogonium bisserata Desvaux (Légumineuse), Digitaria horizontalis Willdenow, (Poaceae) ou de repousses Rauvolfia vomitoria Afzel (Apocynaceae) et Alchornea cordifolia (Schum\& Thonn.) Müll. Arg.(Euphorbiaceae), Bambusa vulgaris Schrad (Poaceae). Tous les 20 types biologiques ont été observés dans la flore étudiée. Les plus dominants sont en nombre d'espèces décroissant: les Thérophytes $(26,3 \%)$, les Nanophanérophytes $(15,3 \%)$, les lianes Microphanérophytes (3,2\%) et les Chaméphytes (8,9\%). Ces types biologiques représentent $54,8 \%$ de la flore (Tableau 1). Les adventices rencontrées sont majoritairement des espèces Guinéo-Congolaises et Soudano-Zambésiennes (122 espèces CG-SZ) contre 92 espèces Guinéo-Congolaises (GC) ou de forêt dense et seulement 4 espèces de savane ou espèces SoudanoZambéziennes (SZ). Plusieurs espèces d'adventices des bananeraies sont des Héliophytes (53 espèces). II existe aussi 8 espèces de lianes pourvues de vrilles, 3 espèces cauliformes, 2 espèces arénicoles et 2 espèces arbustives munies de contreforts. II s'y trouve également, des cas assez rarissime d'espèce munie de racines adventives ou de racines échasses ou encore de plante ripicole (une espèce dans chaque cas). Les courbes de variation du nombre d'espèces adventices en fonction des classes de fréquences relatives des différentes plantations ont-elles une forme unimodale en " $\mathrm{J}$ 》 renversé et les valeurs des coefficients de similarité calculées sont comprises entre $50,1 \%$ et $62,3 \%$.

Tableau1 : Répartition du nombre d'espèces adventices en fonction des types biologiques

\begin{tabular}{|l|l|l|}
\hline Types biologiques & Nombre d'espèces & Espèces (\%) \\
\hline Thérophyte (TH) & 74 & 26,3 \\
Nanophanérophyte (np) & 43 & 15,3 \\
Liane mésophanérophyte (Lmp) & 37 & 13,2 \\
Microphanérophyte (mp) & 35 & 12,4 \\
Chaméphyte (Ch) & 25 & 8,9 \\
Hémicriptophyte (H) & 19 & 6,8 \\
Mésophanérophyte (mP) & 14 & 4,9 \\
Géophytes rhizomateux (Gr) & 9 & 3,2 \\
Hydrophyte nageant (Hydn) & 6 & 2,1 \\
Mégaphanérophyte (MP) & 5 & 1,8 \\
Semi-Aquatique (Se-Aq) & 4 & 1,4 \\
Stolonifère (Sto) & 2 & 0,7 \\
Epiphyte (Ep) & 1 & 0,3 \\
Géophytes (G) & 1 & 0,3 \\
Hydrophyte (Hyd) & 1 & 0,3 \\
Rhizomateux (rh) & 1 & 0,3 \\
Se-Epiphyte (Se-Ep) & 1 & 0,3 \\
Liane mégaphanérophyte (LmP) & 1 & 0,3 \\
Liane nanophanerophyte (Lnp) & 1 & 0,3 \\
hémicriptophyte pyrophytique (Hpy) & 1 & 0,3 \\
\hline Total / Moyenne & 281 & 4,97 \\
\hline
\end{tabular}

Fréquence relative des adventices : Le dénombrement des adventices en fonction des classes de fréquences indiquent qu'en moyenne, seulement une espèce évolue sur la presque totalité des parcelles (81 à 100\%), tandis 
que 10 autres espèces sont présentes sur au moins la moitié des parcelles de bananiers (61 à $80 \%)$. Douze autres sont observées sur 41 à $60 \%$ des parcelles. En somme, 23 espèces adventices sont plus fréquentes et
42 autres espèces sur moins de la moitié des parcelles (21 à 41\%). Elles appartiennent aux classes de fréquences II, III, IV et $V$ et constituent le noyau floristique de base (Tableau 2).

Tableau 2 : Répartition du nombre d'espèces en fonction des classes de fréquences

\begin{tabular}{|l|ll|ll|ll|ll|ll|l|}
\hline \multicolumn{1}{r|}{ Classes } & I & & II & & III & & IV & & V & & \\
Sites & Fa & Pc. & Fa & Pc. & Fa & Pc. & Fa & Pc. & Fa & Pc. & Nombre total d'espèces \\
\hline Site 40 & 138 & 88,5 & 9 & 5,7 & 5 & 3,2 & 3 & 1,9 & 1 & 0,6 & 156 \\
Site 66 & 120 & 88,9 & 12 & 8,9 & 1 & 0,7 & 2 & 1,5 & 0 & 0 & 135 \\
Agnéby & 150 & 90,3 & 10 & 6 & 3 & 1,8 & 2 & 1,2 & 1 & 0,6 & 166 \\
N'gatty & 103 & 85,8 & 11 & 9,2 & 3 & 2,5 & 3 & 2,5 & 0 & 0 & 120 \\
\hline Moyenne & 127,5 & 88,4 & 10,5 & 7,4 & 3 & 2 & 2,5 & 1,8 & 0,5 & 0,3 & 144 \\
\hline
\end{tabular}

( $\mathrm{I} ; \mathrm{II}$...V sont les classes de fréquences selon RAUNKIER, 1905; Fa : la fréquence absolue des espèces et Pc : le pourcentage du nombre d'espèces de la classe par rapport au nombre total d'espèces adventices observées)

Catégories de mauvaises : Les adventices majeures des plantations de bananiers de la zone de Dabou sont au nombre de 8 dont 5 sont majeures sur l'ensemble des 4 plantations et 3 sur seulement un site. Les 5 espèces de mauvaises herbes majeures du milieu étudié sont par ordre décroissant de leur fréquence relative: Cleome ciliata, Panicum laxum, Phyllanthus amarus, Spermacoce latifolia et Phyllanthus urinaria. Ageratum conyzoides, Mariscus cylindristachyus et Cyperus sphacelatus. Alchornea cordifolia, Nymphaea lotus, Digitaria horizontalis, Mariscus cylindristachyus, Ageratum conyzoides et Phyllanthus amarus sont les 6 adventices majeures potentielles de la zone de Dabou. Les adventices accompagnatrices sont au nombre de 15 espèces dans la zone d'étude. Dans l'ordre décroissant de leur fréquences relatives: Salvinia nymphellula, Struchium sparganophora, Pityrogramma calomelanos, Fimbritylis littoralis, Passiflora foetida, Ludwigia erecta, Euphorbia prostrata, Pentodon pentandrus, Nephrolepis bisserata, Emilia praetermissa, Bambusa vulgaris, Glynus pilosa, Chromoleana odorata, Vernonia cinerea et
Clidemia hirtum. Les adventices accidentelles ou locales des bananeraies sont plus nombreuses 106 espèces en moyenne et ont des fréquences relatives très faibles. II s'agit entre autre de Rauvolfia vomitoria, Amaranthus viridis, Panicum brevifolium, Lygodium microphyllum, Baphia nitida, Paullina pinnata, Trianthema portulacastrum, Solanum rugosum, Citrilus lanatus, etc. Abondance-dominance des mauvaises herbes: En moyenne, aucune mauvaise herbe n'a été observée avec un indice d'abondance-dominance moyenne égal à 4 sur la zone d'étude. Les mauvaises herbes abondantes et à fortes densités ne sont présentes que sur certaines parcelles. Quatre espèces de mauvaises herbes sont assez abondantes et ont des densités moyennes sur la zone étudiée: Cleome ciliata, Spermacoce latifolia, Panicum laxum et de Phyllanthus urinaria (Tableau 3). La première espèce est la seule qui soit assez abondante avec une densité moyenne sur tous les sites. Les espèces peu agressives sont au nombre de 112 en moyenne par site.

Tableau 3 : Valeurs des paramètres floristiques moyennes des adventices assez abondantes sur l'ensemble des 4 bananeraies

\begin{tabular}{|l|l|l|l|}
\hline Espèces & Frm (\%) & CSF(e) & IADm \\
\hline Cleome ciliata & 75,6 & 6,5 & 3 \\
\hline Spermacoce latifolia & 53,6 & 5,2 & 3 \\
\hline Panicum laxum & 68,1 & 5,6 & 3 \\
\hline Phyllanthus urinaria & 59,8 & 5,2 & 3 \\
\hline
\end{tabular}

Frm : Fréquence relative Moyenne, $\operatorname{CSF}(\mathrm{e})$ : coefficient de Contribution floristique ; IADm : Indice d'abondance-dominance moyen

Études phytoécologiques

Comparaison des plantations à partir des variables :

L'observation des résultats de l'ensemble des sites indique que les 4 plantations sont différentes les unes des autres du point de vue des valeurs des 18 variables agrotechniques, naturelles et floristiques choisies (Figure 
3). Les deux principaux axes $F 1(60,1 \%)$ et $F 2(30,26 \%$.) renferment $90,36 \%$. de la variabilité et séparent aisément les 4 plantations l'une de l'autre. La plantation d'Agnéby diffère des autres par des périodes moyennes d'observation et de traitements herbicides (Tao et $\mathrm{TaH}$ ) assez longues (14 et 21 semaines), le nombre moyen d'espèces adventices par placette (Nes / p) plus élevé (13,5 espèces par placette), le nombre moyen d'échantillons relativement élevé (11,7 échantillons par parcelle) et par un type de sol particulièrement argileux. N'gatty se distingue des autres plantations par la position des parcelles en milieu de pente en moyenne comme à Agnéby contrairement aux sites 40 et 66 où les parcelles sont en moyenne en haut de pente et également par des fréquences relatives moyennes des adventices assez élevées $(30,6$ p.c.). Le site 40 se caractérise par des parcelles plus âgées ( 3 ans et demi en moyenne) que celles des autres plantations, un nombre relativement plus important de bananiers par parcelle (7353), des indices d'abondance-dominance globaux plus importants (supérieurs à 2 en moyenne) des parcelles plus vastes (3,9 ha en moyenne) et plus recouvertes par les adventices $(57,6 \%)$. Ce dernier paramètre le rapproche des parcelles d'Agnéby recouvertes à $55,6 \%$ par les mauvaises herbes et l'oppose au site 66 et à la plantation de N'gatty dont les recouvrements globaux des mauvaises herbes sont inférieurs en moyenne à $50 \%$. Par ailleurs, le site 66 se distingue des autres par un taux de coupe relativement plus important $(55,2 \%$ en moyenne) et un recouvrement assez important des bananiers $(59,5 \%$. en moyenne). Cette dernière valeur la rapproche cependant du site 40 dont le recouvrement des bananiers ( $66,4 \%$ en moyenne), l'indice d'abondancedominance global moyen des adventices et l'âge moyen des parcelles sont plus élevés.

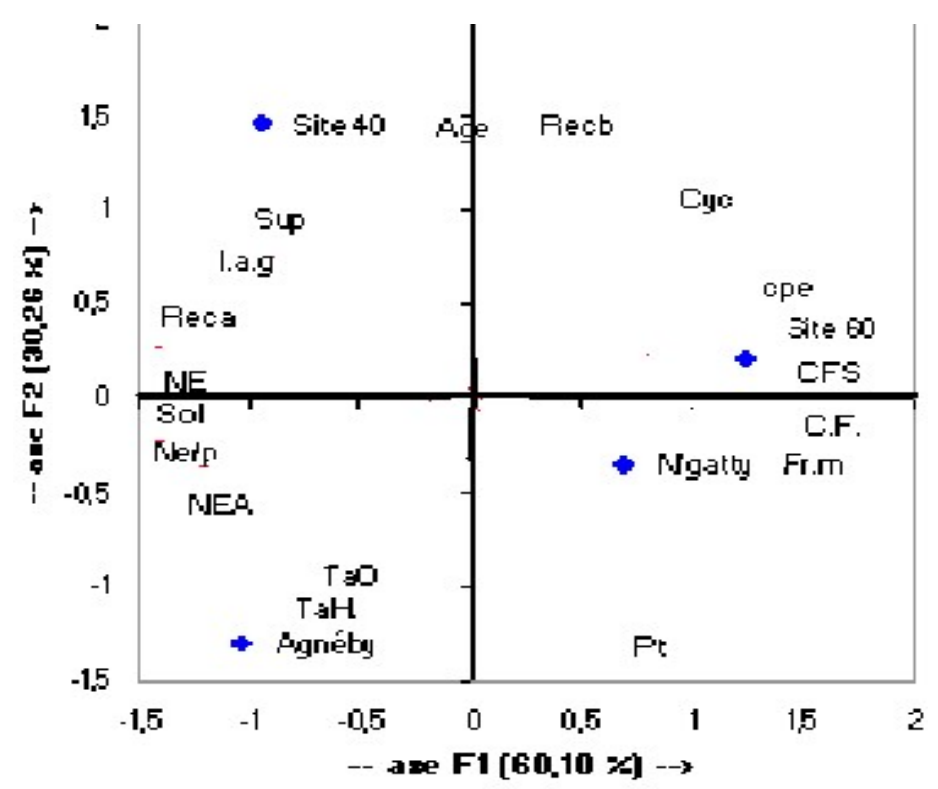

Figure 3-- Discrimination des quatre bananeraies à partir des variables floristiques et écologiques. Les variables agrotechniques : Sup =la superficie; NE=le nombre d'échantillons; $\mathrm{Pt=la}$ position dans la toposéquencem; Sol=le type de sol; Cyc=le cycle; Age=l' âge de la parcelle; TaH=le temps entre deux traitements herbicides successifs; Tao=le temps entre la date d'observation et la date d'application herbicide; Recb=le recouvrement des bananiers; Cpe=le taux de bananiers coupés; NEA=le nombre d'espèces adventices; Reca=le recouvrement des adventices; Nes/p=le nombre d'espèces adventices par placette; C.F.=la contribution floristique; I.a.g=l'indice d'abondance-dominance global des adventices; Fr.m=la fréquence relative moyenne des adventices et CFS=la contribution floristique spécifique moyenne des adventices.

Relations entre les variables: L'analyse en composantes principales a permis de discriminer les 18 variables. L'axe F1 est lié au recouvrement des bananiers (Recb) et l'axe F2 à la contribution floristique (CF) et à la contribution spécifique $(\operatorname{CSF}(e))$ des espèces adventices.
La corrélation est par conséquent significativement très faible entre Recb (Recouvrement des bananiers) et CF (Contribution Floristique, $r=0,11$ ) et entre Recb et $\operatorname{CSF}(e)(r=0,15)$. En outre, les axes F1 et F2 permettent de définir 4 groupes de variables. Le premier est formé 
par le recb, Cyc (Cycle des bananiers), Cpe (taux de coupe) CF, et CSF (Contribution Floristique Spécifique) qui sont positivement corrélées entre elles. La contribution des adventices à la végétation n'est donc pas liée au recouvrement des bananiers. Les liens entre le recouvrement des bananiers sont moyens avec le cycle $(r$ $=0,49)$ et la coupe $(r=0,41)$. Le deuxième groupe est constitué des variables: Age, nombre de bananiers (Npb), Superficie (Sup), Indice d'abondance-dominance global des adventices (lag), recouvrement global des adventices (Reca), et le nombre d'échantillons (NE). Ces paramètres sont positivement et fortement corrélés $(0,47$ $<r \leq 1)$. Le troisième groupe comprend les variables : sol, périodes de traitement herbicide et d'observation $(\mathrm{TaH}$,

\section{DISCUSSION}

La flore des quatre bananeraies est très diversifiée. Elle est composée de 281 espèces identifiées. Cette diversité végétale s'observe par la présence de Dicotylédones, de Monocotylédones, de Bryophytes et de Ptéridophytes. Chacune de ces classes est représentée par plusieurs genres et plusieurs espèces notamment, les Dicotylédones. La prédominance des espèces de cette dernière classe par rapport à celle des autres classes semble être une caractéristique de la zone étudiée. Ainsi,La flore hypogée des plantations de palmiers à huile étudiées à Dabou était dominée à $64 \%$ par les Dicotylédones (Traoré et al., 2007). Ce résultats se rapprochent des $83 \%$ de Dicotylédones observées dans la Région de Gagnoa en culture de maïs (Kouadio, 2003) et des $75,81 \%$ d'espèces de cette classe dans la flore adventices des ananeraies de Bonoua et de N'douci. De plus, la présence de nombreuses espèces de forêt indique que la flore étudiée a les caractéristiques d'une zone forestière. La présence des espèces de savane pourrait s'expliquer par le déplacement de sol pour la réalisation des routes en provenance des savanes intruses de la région. Par ailleurs, la flore est dominée par 5 familles, principalement par les Poaceae, les Cyperaceae, les Euphorbiaceae, les Rubiaceae et les Asteraceae. Ces familles sont également, celles qui dominent la flore étudiée dans la même zone (Traoré et al., 2007). Ces informations sont indicatrices d'une zone forestière fortement dégradée, la végétation étant dominée par les Thérophytes, les Nanophanérophytes, les Microphanéphytes et les Chaméphytes. Les individus appartenant aux espèces de Mésophanérophytes et de Mégaphanérophytes observés dans la végétation sont au stade de jeunes plants. Cette flore contient tout de même deux espèces ivoiriennes, rares et menacées d'extinction (Kouadio,2010). Il s'agit de Leptoderris miegei Aké-Assi \&
Tao), Nombre d'Espèces Adventices (NEA) et Nombre d'espèces par placette (Nes / p) qui sont également, positivement corrélées. Le quatrième groupe est formé de la Position topographique $(\mathrm{Pt})$ et de la Fréquence relative moyenne des adventices (Frm). Elles sont positivement liées $(r=0,55)$. Ce qui indique que les mauvaises herbes sont plus présentes sur les parcelles de bas et de milieu de pente que sur celles situées en haut de pente. En outre, les variables $\mathrm{TaH}$ et $\mathrm{TaO}, \mathrm{Nbp}$ et Sup sont très positivement liées. De même, l'indice d'abondancedominance global moyen des adventices et l'âge des parcelles sont positivement liés. Par contre, l'âge des parcelles et le cycle des bananiers le sont faiblement.

Mangenot (Fabaceae) et Milletia takou Lorougnon (Fabaceae) (UICN, 2008). Le milieu étudié est favorable à l'évolution de plusieurs espèces appartenant à des genres et à des familles très variés. De la sorte, il n'existe véritablement pas de grande famille ou de grand genre multispécifique. Cette observation est caractéristique de la flore ivoirienne, très diversifiée où on note la prépondérance de familles à faible effectif (Aké-Assi, 2002). Par ailleurs, certaines espèces considérées comme des pestes pour d'autres cultures et dans d'autres climats semblent être maîtrisées dans le milieu étudié. C'est le cas de Euphorbia hirta, peste du cotonnier au Centre et au Nord de la Côte d'Ivoire ou de Cyperus esculentus, C. rotundus, Chromolaena odorata, Echinocloa crus-pavonis, E. colona, E. pyramidalis et d'Imperata cylindrica (Johnson, 1997; Ipou, 2000 ; Boraud, 2000 ; Touré 2009). Cela pourrait s'expliquer par le climat et les techniques culturales (forte pluviométrie, traitements herbicides répétés). Cependant la présence de Croton hirtus dans cette flore devrait inspirer de la vigilance du fait qu'elle est envahissante en culture de manioc et de maïs dans la zone d'étude et que par ailleurs elle résiste aux herbicides usuels à la dose utilisée. La phénologie des adventices montre que la périodicité des traitements herbicides est assez favorable à la reproduction des mauvaises herbes, particulièrement des 8 espèces majeures de la zone. Les traitements herbicides se pratiquent au-delà des 8 semaines préétablies dans le calendrier cultural. De plus, le nombre d'applications annuelles d'herbicides est de 4 contre 6 en théorie. Aussi l'utilisation d'une seule matière active (le Glyphosate) à des doses parfois inférieures à celle recommandée, souvent dans des conditions météorologiques peu certaines avec la présence des vents et des pluies peut être à la base de nombreux cas 
de résistances par accoutumance observés au niveau des adventices. Cette observation est conforme à celle faite par le CIRAD (2001). Parmi les espèces d'adventices recensées, 8 sont également des hôtes de nématodes. Il s'agit de Ageratum conyzoides, Commelina benghalensis, Linderia diffusa, Cyperus sphacelatus, Oldenlandia corymbosa, Phyllanthus urinaria et Vernonia cinerea (Tano, 2005).

L'analyse en composantes principales des variables agronomiques et floristiques a permis d'établir une corrélation entre la période d'observation et celle des traitements herbicides. Cette relation s'explique par le fait que les observations sont réalisées un ou deux jours avant l'application des herbicides. Aussi la synergie entre le peuplement des bananiers et la superficie des parcelles traduit-elle le calcul des densités effectué avant la mise en plantation des parcelles. Les bananiers sont plantés à $2 \mathrm{~m}$ sur la ligne et 1,8 $\mathrm{m}$ entre les lignes de sorte à obtenir une densité de 1850 pieds par hectare. En outre, le faible lien entre l'âge de la parcelle et le cycle cultural des bananiers indique l'existence des problèmes de fertilité des sols, d'œilletonnage, d'haubanage, de coups de vent, de pourriture racinaires et d'inondation qui occasionnent des replantations partielles ou totales, voire la mise en jachère des parcelles. Ces problèmes contrarient souvent l'évolution linéaire du cycle des bananiers par rapport à l'âge des parcelles telle que prévue par la succession culturale par les rejets-fils.

La relation entre le sol, la position des parcelles dans la toposéquence et l'enherbement est due à la nature, au mode de dissémination des semences des adventices, à la fertilité du sol et surtout à l'eau. Ces facteurs sont très discriminants vis-à-vis de la flore adventice. Les parcelles étudiées sont très humides du fait de l'irrigation régulière et de la proximité de la nappe phréatique. L'incidence de ces facteurs sur la flore a été observée en riziculture (Johnson, 1997) et en maïsiculture (Kouadio, 2003) dans la région de Gagnoa. De plus d'autres études montrent que les semences de mauvaises herbes colonisent abondamment les 10 et 20 premiers $\mathrm{cm}$ du sol (De Row et Van Oers, 1988 ; Donfack, 1993 ; Traoré et al., 2007). D'autres travaux ont montré que les sols des plateaux (1112 jeunes plants) sont plus riches en graines de mauvaises herbes que les parcelles en milieu de pente (1085 jeunes plants) et celles de bas de pente avec 491 jeunes plants (Traoré, 2000). Par ailleurs, il existe un lien positif entre l'âge des parcelles et l'indice d'abondancedominance des adventices. Cette relation indique l'enrichissement continu des sols des bananeraies en semences de mauvaises herbes. Ces semences sont produites localement par les plantes mères et sont stockées dans le sol d'où elles germeront en temps favorables. Beuret (1980) affirmait à ce sujet qu'en l'absence de labour, le stock semencier du sol issu des Poaceae est abondant. Ceci est une explication à la présence de la trentaine d'espèces de Poaceae rencontrées sur les parcelles. Aussi, l'opposition entre certains paramètres du milieu tels que la position dans la toposéquence, le taux de coupe, le recouvrement, le nombre de pieds, le cycle des bananiers et les variables floristiques indique la possibilité de contrôler l'enherbement en agissant sur ces variables du milieu. Une coupe bien programmée depuis l'œilletonnage permet de maintenir en permanence, un taux de recouvrement important des bananiers et de réduire ainsi les levées, le recouvrement, l'indice d'abondancedominance et la fréquence des mauvaises herbes. Ce qui permet l'augmentation de la période des applications herbicides et la réduction de la quantité d'herbicides utilisés sur les parcelles. Cette influence des paramètres écologiques a été observée sur la flore des rizières (Johnson, 1997).

Les périodes de traitements herbicides sont relativement plus longues à N'gatty et à Agnéby. Cette observation s'explique par le sol très argileux de ces deux plantations mais aussi par une technique de paillage différente des autres sites. Sur ce site, particulièrement à N'gatty, le drainage des eaux a conduit à réduire de moitié la surface des planches, de sorte que le paillage de ces surfaces par les feuilles de bananiers est presque total. De même, le paillage par pied de bananier est défavorable à l'enherbement des parcelles. Ce qui permet de réduire le nombre de traitements herbicides. L'enherbement plus important des parcelles du site 40 et des parcelles d'Agnéby est essentiellement lié au sol et à l'humidité des parcelles. Ces sols sont en majorité la Tourbe et les Argiles, riches azotes, en potassium en phosphore et en ologoéléments. En outre, le cycle, la coupe, le recouvrement et le nombre de bananiers sont des facteurs inhibiteurs de la flore. Ils exercent également un effet de sélection dû essentiellement à l'ombrage et au pédoclimat dont ils sont à l'origine au niveau de la parcelle. La sélection de la flore peut également s'expliquer par l'inefficacité de la matière active due à l'emploi des mêmes herbicides par effet d'accoutumance. II apparaît dans ces conditions des peuplements monospécifiques comme c'est le cas, ici, où la flore est dominée par 8 espèces de mauvaises herbes qui opposent une résistance phénologique aux herbicides principalement. Aussi distingue-t-on des espèces tolérantes aux herbicides usuels c'est-à-dire des espèces d'adventices ne faisant pas partie du spectre d'efficacité 
des herbicides utilisés. De plus, le non respect du calendrier du désherbage chimique s'explique ici par le coût élevé de ces intrants (37 423,08 CFA an moyenne

\section{CONCLUSION}

La flore des bananeraies étudiées comprend 281 espèces d'adventices reparties entre 222 genres et 83 familles. Les Dicotylédones sont les plus nombreuses. Cette classe renferme 64 familles, 165 genres et 199 espèces adventices. Les Monocotylédones sont représentées par 8 familles, 43 genres et 67 espèces qui représentent des espèces adventices observées. Huit familles de Bryophytes sont également présentes et se répartissent entre 10 genres et 11 espèces. Elle devance de peu les Ptéridophytes qui sont représentées par 3 familles botaniques regroupant 4 genres et 4 espèces. Les familles sont souvent représentées par 2 espèces végétales au plus. Les familles dominantes sont dans l'ordre décroissant les Poaceae, Cyperaceae, les Euphorbiaceae, les Rubiaceae et les Asteraceae. Par ailleurs, un nombre relativement élevé d'espèces de champignons, de mousses et d'algues, non identifiées et évoluant sous les bananiers poussent sur divers substrats (organes végétaux en décomposition, fumier, sol).

L'étude de Phytosociologie révèle que 8 espèces végétales sont majeures dans la zone de Dabou. Ces mauvaises herbes sont plus fréquentes, très agressives vis-à-vis des bananiers et aussi assez abondantes avec des indices d'abondance-dominance supérieurs ou égaux à 3 malgré l'usage des herbicides. Ces informations démontrent l'efficacité des herbicides à contrôler un grand nombre d'espèces d'adventices tout en maintenant les espèces potentiellement majeures ou comme des espèces accompagnatrices ou mineures. Le fait que 8 espèces soient majeures malgré l'usage des herbicides démontre l'inefficacité de ces produits à contrôler

\section{REMERCIEMENTS}

Nous remercions le Professeur Laurent Aké-Assi pour sa collaboration scientifique qui a permis l'identification des espèces végétales et le Centre National de Floristique de Côte d'Ivoire pour avoir servir de cadre à la rédaction de

\section{REFERENCES}

Adé J, 2006. Le marché européen se ferme. Frat-Mat Agri, Abidjan, Côte d'Ivoire $3: 40$.

Aké-Assi L, 2001. Flore de la Côte d'lvoire : catalogue systématique, biogéographie et écologie, Mémoire de botanique systématique, tome 1 , Boissiera 57: 396. par hectare). Cette situation a déjà été signalée par d'autres auteurs (Johnson, 1997 ; Kouadio, 2003).

durablement ces dernières espèces adventices dans les conditions de culture actuelles. La dynamique posttraitement herbicide des adventices démontre le rétablissement de la végétation au bout de 4 à 6 semaines. II apparaît, ici, une résistance phénologique et physiologique des espèces végétales au Glyphosate. II faut par conséquent envisager d'autres pistes, notamment la lutte biologique (Kouadio, 2009).

L'étude Phytoécologique indique que les variables agronomiques choisies, notamment, le type de sol, la position dans la toposéquence, de l'âge et de la superficie des parcelles ont effectivement une incidence directe sur l'évolution des adventices. En outre, la présence de certaines adventices peut aussi se justifiée par la mauvaise préparation de terrain avant la mise en culture (absence de labour sur certaines parcelles) et également par une négligence des travailleurs qui consomment certains fruits et laissent tomber les semences dans les plantations. C'est le cas des papayers (Carica papaya L. Caricaceae), des goyaviers (Pissidium goyava L. (Myrtaceae), de Corossoliers (Annona muricata L., Annonaceae), des manguiers (Mangifera indica L. Anarcadiaceae), des orangers (Citrus sinensis L, Rutaceae.) et même des cacaoyers (Theobrama cacao L., Sterculiaceae) observés accidentellement en plantation de bananiers. Dans d'autres circonstances, c'est la pratique agronomique qui est responsable de la présence des mauvaises herbes. C'est par exemple, le cas de Bambusa vulgaris, qui est souvent coupé frais et plantés pour être utilisé au moment de l'haubanage. Plus tard, l'humidité du sol aidant, la plante reprend et devient une mauvaise herbe.

la thèse. Nos remerciements vont également à l'endroit de la Compagnie des Bananes de Côte d'Ivoire pour les infrastructures et le soutien financier.

Aké-Assi L, 2002. Flore de la Côte d'Ivoire : catalogue systématique, biogéographie et écologie, Mémoire de botanique systématique, tome 2, Boissiera 58 : 401.

Aman KG, 1978. Flore et végétation des adventices dans l'hévéaculture en basse Côte d'Ivoire, Station 
expérimentale de I'IRCA. Etude écologique: Dynamique et structure. Thèse de Spécialité : Ecologie végétale, Université de CocodyAbidjan, Côte d'Ivoire.

Beuret E, 1980. Influence de la monoculture et des méthodes de travail du sol sur la flore adventice et le stock grainier du sol. COLUMA $1980 ; 2$ : 389-99.

Boraud NKM, 2000. Etude floristique et phytoécologique des adventices dans les complexes sucriers de Ferké1 et 2, de Borotou-Koro et de Zuenoula, En Côte d'Ivoire. Thèse de doctorat $3^{\mathrm{e}}$ cycle, Université de Cocody-Abidjan, Côte d'Ivoire.

Braun-Banquet J, 1932. Plant sociology. The study of plant community (English translation of "Pflanzensoziologie" by G.D. Fuller and H.S. Conard). Univesity of Chicago.

BCEAO, 2008. Indicateurs économiques, Direction nationale pour la Côte d'lvoire, Agence principale d'Abidjan, Service des études statistiques, Abidjan, Côte d'Ivoire.

Chicouène $D, 2000$. Evaluation du peuplement de mauvaises herbes en végétation dans une parcelle : II-Protocoles rapides pour un usage courant. Phytoma-Défense des cultures 524 : 18-23.

CIRAD, 2001. Place des herbicides dans l'itinéraire technique, Malherbologie tropicale WWW.cirad.fr, décembre 2008.

Daget P. et Poissonnet J, 1969. Analyse phytosociologique des prairies: applications agronomiques. Docum. CNRS.-CEPE.Montpellier-France.

De Row A. et Van Oers C, 1988. Seeds in soil and their relation to shifting cultivation in the Ivory Coast. Weed Research, 28: 373-381.

Donfack P, 1993. Etude de la dynamique de la végétation après abandon de la culture au NordCameroun. Thèse de doctorat, Université de Yaoundé, Cameroun.

Emberger, 1966. Réflexion sur les spectres biologiques de Raunkiaer. Mém. Coc. Bot. Fr., 148-156.

Farcey P, 2008. La banane antillaise, Univers Nature, 2 p, WWW.univers. nature.com, 12 déc 2008.

Guinochet M, 1973. Phytosociologie, Masson et Cie, Paris, France.

Goldsmith EB, Hildyard P, McCully P, 1990. Terre arable In: 5000 jours pour sauver la planète : 96-115. Édition Chêne, Barcelone, Espagne.
Godron M, 1968. Quelques applications de la notion de fréquence en écologie végétale. Oecol. Plant., $3: 185-212$.

Gounot M, 1969. Méthodes d'études quantitatives de la végétation, Masson, Paris, France.

Ipou IJ, 2000. Importance de Euphorbia heterophylla L. (Euphorbiaceae) dans la végétation adventice dans les systèmes culturaux à base de cotonniers dans le Worodougou, en Côte d'Ivoire. D.E.A. de Botanique, option Ecologie Végétale, Université de Cocody, Abidjan, Côte d'Ivoire.

Ipou IJ, 2005. Biologie et écologie de Euphorbia heterophylla L. (Euphorbiaceae) en culture cotonnière, au nord de la Côte d'Ivoire. Thèse de l'Université de Cocody, Abidjan, Côte d'Ivoire.

Johnson DE, 1997. Les adventices en riziculture en Afrique de l'Ouest. Weeds of rice in West Africa, ADRAO / WARDA, Bouaké, Côte d'Ivoire.

Kouadio YP, 2003. Dynamique des adventices dans la culture de maïs de second cycle, dans la région de Gagnoa (Côte d'Ivoire), D.E.A. de Botanique, Laboratoire de Botanique, U.F.R. Biosciences, Université de Cocody-Abidjan, Abidjan Côte d'Ivoire

Kouadio YP, 2009. Contrôle de l'enherbement des bananeraies industrielles par des Légumineuses de couverture en basse Côte d'lvoire, Agronomie africaine, 20 (3):17-20.

Kouadio YP, 2010. Utilisation des plantes de couvertures comme moyens de lutte biologique contre la flore adventice des bananeraies industrielles de Dabou au Sud de la Côte d'Ivoire. Thèse Unique de Doctorat, Université Félix HOUPHOUET BOIGNY, Abidjan, Côte d'Ivoire.

Le Bourgeois T, 1993. Les mauvaises herbes dans la rotation cotonnière au Nord du Cameroun. Thèse de Doctorat, Université de Montpellier II, France.

Le Bourgeois T, Le Breton G, Grillet N, Chiroleu F, 2004. Caractérisation des enherbements en culture de canne à sucre à la Réunion. 19ème conférence internationale du Columa, Dijon, France, AFPP. 8 pages.

Le Breton G, et Le Bourgeois T, 2005. Analyse comparée de la flore en culture d'ananas et de canne à sucre à la Réunion. Pdf consulté le 19 avril 2007. http://www.prpv.org/index.php/fr/content/downlo ad/1586/12827file/Le\%20rapport\%20anana. 
Lebrun J, 1966. Les formes biologiques dans les végétations tropicales. Bull. Soc. Bot. France: 164-175.

Le Touzel R, 1985. Carte phytogéographique du Cameroun (au 1/500000, 1). Domaine sahélien et soudanien. IRA (Herbier national) Yaoundé Institut de la Carte internationale de la Végétation, Toulouse : 1-26.

Loudyi MC, 1985. Etude botanique et écologique de la végétation du plateau de Meknès (Maroc). Thèse, USTL, Montpellier, France.

Maillet J, 1981. Evolution de la flore adventice dans le montpellierais sous la pression des techniques culturales. Thèse DDI, USTL, Montpellier, France.

Marnotte $P, 1989$. Plante nuisible, désherbage, herbicide, au complexe sucrier de la SOSUCO. Rapport de mission de Malherbologie à Banfora (BurkinaFaso).

Ministère de l'économie et des finances, 2007a. Indicateurs conjoncturels, bulletin trimestriel Direction Générale de l'économie, Direction de la conjoncture et des prévisions économiques $43: 30$.

Ministère de l'économie et des finances, 2007b. Indicateurs conjoncturels bulletin trimestriel, Direction Générale de l'économie, Direction de la conjoncture et des prévisions économiques (45) : 44.

Ministère de l'économie et des finances, 2007c. La Côte d'Ivoire en chiffre éd. 2007, Direction Générale de l'économie : 44-94.

Ministères des Affaires Etrangères, CIRAD, GRET, 2006. Memento de l'agronome, Paris, France.

Raunkiaer S, 1934. The life forms of plants and statistical plant geography. Clarendon Press. Oxford.

Sørensen T, 1948. A method of establishing groups of equal amplitude in plant sociology based on similary of species content. Det kongelige Danske Videns kaherness selskab. Biologiske Skrifter, 5, 4: 1-34.

Tano EKJ, 2005. Relation entre les mauvaises herbes et les nématodes en culture de bananier plantain (Musa spp.) à forte densité, D.E.A. de Botanique, option Ecologie Végétale, Laboratoire de Botanique, U.F.R. Biosciences, Université de Cocody, Abidjan, Côte d'Ivoire, $41 p$.

Touré A, 2009. Diversité floristique et degré d'infestation des mauvaises herbes des agroécosystèmes environnant la forêt classée de Sanaimbo, dans le centre-est de la Côte d'Ivoire, Thèse Unique de Doctorat de I'Université de Cocody-Abidjan, Côte d'Ivoire.

Traoré $H, 1991$. Influence des facteurs agro-écologiques sur la constitution des communautés adventices des principales cultures céréalières (sorgho, mil, maïs) du Burkina Faso. Thèse de doctorat, Montpellier, France.

Traoré K, 2000. Etude quantitative des stocks de semences d'adventices des sols rizicoles de la station de l'Association pour la riziculture en Afrique de l'Ouest (A.D.R.A.O.) à Bouaké (Côte d'Ivoire). D.E.A. de Botanique, option Ecologie Végétale, Laboratoire de Botanique, U.F.R. Biosciences, Université de Cocody-Abidjan, Abidjan, Côte d'Ivoire.

Traoré K, Ballo B, Pene CB, Aké S, 2007. Caractérisation de la flore adventice hypogées dans les agroécosystèms du palmier à huile (Elaeis guinéensis Jacq.) en basse Côte d'Ivoire : cas de La mé et de Dabou, Agronomie Africaine 19 (3) : 289-299.

UICN, 2008. Liste rouge des espèces végétales menacées d'érosion génétique.

http://www.planetoscope.com/fruits-legumes/1177production-mondiale-de-bananes.html

Avenard J.M., Eldinn M., O. Girard, J. Sircoulon, Touchebeuf P.,Adjanohoun E. Perraud A.,1971 Le milieu Naturel de Côte d'Ivoire, ORTOM, 68 p. 